\title{
Establishment of Local Diagnostic Reference Levels of Pediatric Abdominopelvic and Chest CT Examinations Based on the Body Weight and Size in Korea
}

\author{
Jae-Yeon Hwang ${ }^{1}$, Young Hun Choi ${ }^{2}$, Hee Mang Yoon ${ }^{3}$, Young Jin Ryu ${ }^{4}$, Hyun Joo Shin ${ }^{5}$, Hyun Gi Kim ${ }^{6}$, \\ So Mi Lee ${ }^{7}$, Sun Kyung You ${ }^{8}$, Ji Eun Park ${ }^{9}$ \\ ${ }^{1}$ Department of Radiology, Research Institute for Convergence of Biomedical Science and Technology, Pusan National University Yangsan Hospital, \\ College of Medicine, Pusan National University, Yangsan, Korea; ${ }^{2}$ Department of Radiology, Seoul National University Hospital, Seoul National \\ University College of Medicine, Seoul, Korea; ${ }^{3}$ Department of Radiology and Research Institute of Radiology, University of Ulsan College of \\ Medicine, Asan Medical Center, Seoul, Korea; ${ }^{4}$ Department of Radiology, Seoul National University Bundang Hospital, Seoul National University \\ College of Medicine, Seongnam, Korea; ${ }^{5}$ Department of Radiology and Research Institute of Radiological Science, Severance Children's Hospital, \\ Yonsei University College of Medicine, Seoul, Korea; ${ }^{6}$ Department of Radiology, Eunpyeong St. Mary's Hospital, College of Medicine, The Catholic \\ University of Korea, Seoul, Korea; ${ }^{7}$ Department of Radiology, Kyungpook National University Hospital, School of Medicine, Kyungpook National \\ University, Daegu, Korea; ${ }^{8}$ Department of Radiology, Chungnam National University Hospital, Chungnam National University College of Medicine, \\ Daejeon, Korea; ${ }^{9}$ Department of Radiology, Ajou University Hospital, School of Medicine, Ajou University, Suwon, Korea
}

Objective: The purposes of this study were to analyze the radiation doses for pediatric abdominopelvic and chest CT examinations from university hospitals in Korea and to establish the local diagnostic reference levels (DRLS) based on the body weight and size.

Materials and Methods: At seven university hospitals in Korea, 2494 CT examinations of patients aged 15 years or younger (1625 abdominopelvic and 869 chest CT examinations) between January and December 2017 were analyzed in this study. CT scans were transferred to commercial automated dose management software for the analysis after being de-identified. DRLs were calculated after grouping the patients according to the body weight and effective diameter. DRLs were set at the 75th percentile of the distribution of each institution's typical values.

Results: For body weights of $5,15,30,50$, and $80 \mathrm{~kg}$, DRLs (volume CT dose index [CTDI $\mathrm{CTol}_{\text {vol }}$ ) were 1.4, 2.2, 2.7, 4.0, and 4.7 mGy, respectively, for abdominopelvic CT and 1.2, 1.5, 2.3, 3.7, and $5.8 \mathrm{mGy}$, respectively, for chest CT. For effective diameters of $<13 \mathrm{~cm}, 14-16 \mathrm{~cm}, 17-20 \mathrm{~cm}, 21-24 \mathrm{~cm}$, and $>24 \mathrm{~cm}$, DRLs (size-specific dose estimates [SSDE]) were 4.1, 5.0, 5.7, 7.1, and 7.2 mGy, respectively, for abdominopelvic CT and 2.8, 4.6, 4.3, 5.3, and 7.5 mGy, respectively, for chest CT. SSDE was greater than CTDI $_{\text {vol }}$ in all age groups. Overall, the local DRL was lower than DRLs in previously conducted dose surveys and other countries.

Conclusion: Our study set local DRLs in pediatric abdominopelvic and chest CT examinations for the body weight and size. Further research involving more facilities and CT examinations is required to develop national DRLs and update the current DRLs.

Keywords: Diagnostic reference level; Computed tomography; Child; Radiation dose; Radiation protection

\section{INTRODUCTION}

CT is an essential diagnostic modality for medical imaging, and its use has increased dramatically over the past several decades [1-6]. In the United States, according to the Organization for Economic Cooperation

Received: July 13, 2020 Revised: August 11, 2020 Accepted: September 16, 2020

This study was supported by the Dong Kook Life Science. Co., Ltd., Republic of Korea

Corresponding author: Jae-Yeon Hwang, MD, PhD, Department of Radiology, Pusan National University Yangsan Hospital, 20 Geumo-ro, Mulgeum-eup, Yangsan 50612, Korea.

- E-mail: jyhwang79@gmail.com

This is an Open Access article distributed under the terms of the Creative Commons Attribution Non-Commercial License (https:// creativecommons.org/licenses/by-nc/4.0) which permits unrestricted non-commercial use, distribution, and reproduction in any medium, provided the original work is properly cited. 
and Development [1], the number of CT examinations was 78.9 per 1000 people in 1995 and 270.5 per 1000 people in 2018. Furthermore, CT examinations in Republic of Korea have dramatically increased from 74.6 per 1000 people in 2007 to 204.6 per 1000 people in 2017. This trend is also apparent in pediatric CT scans [2,4-6]. According to a recent study analyzing the American College of Radiology dose index registry [5], approximately 126000 single-phase abdominopelvic and 17000 single-phase chest CT scans were performed for pediatric patients between 2011 and 2016, with the number of $\mathrm{CT}$ scans increasing annually.

The radiation dose for pediatric CT scans merits special concern. Children have a greater risk for stochastic effects of radiation compared to adults [7] because of their smaller body habitus, growing body organs that are more radiosensitive than adult tissue, and a longer lifetime of exposure [3]. Therefore, optimization of the pediatric CT examination is crucial in pediatric imaging to achieve an acceptable image quality with appropriate minimal radiation exposure.

The diagnostic reference level (DRL) is a tool for the optimization of $\mathrm{X}$-ray procedures. It was introduced by the International Commission on Radiological Protection (ICRP) in 1996 [8]. Traditionally, age bands have been used to establish DRLs for pediatric X-ray procedures because of their ease of applicability. However, even within each age group, there are considerable variations in the body weight and size, which are more appropriate grouping bases for pediatric CT examinations [9-11].

Before this study, no dose surveys for pediatric body CT examinations had been conducted using CT data from multiple hospitals in Korea. The purposes of this study were to analyze the radiation dose of pediatric body CT examinations from several university hospitals in Korea, to assess the feasibility of using an automated dose management system for the analysis of radiation dosages from multiple hospitals, and to establish the local DRLS for pediatric abdominopelvic and chest CT examinations by grouping patients according to the body weight and size.

\section{MATERIALS AND METHODS}

The Institutional Review Board of each hospital from where CT scans were collected approved this retrospective study (IRB No. 04-2018-018). The requirement for informed consent was waived, as this study showed minimal risk.

\section{Data Collection}

We collected pediatric abdominopelvic and chest CT scans at nine university hospitals from January to December 2017. At least one pediatric radiologist was employed at each hospital. Inclusion criteria were as follows: 1 ) age of 15 years or less; 2) single-phase contrast-enhanced abdominopelvic and chest CT examinations; and 3) CT scans with clinically acceptable image quality, as assessed by the pediatric radiologist at each institution. CT angiography examinations, multiphase CT examinations, CT scans with inadequate image quality for daily practice, and CT examinations performed for research were not included for this study. Exclusion criteria were a lack of information on the body weight, body weight $>80 \mathrm{~kg}$, and dual-energy CT examinations.

The scout image, a set of axial images, and dosage reports were stored in the Digital Imaging and Communications in Medicine (DICOM) format after being de-identified, and data were transferred to the hospitals. Patients' body weights were collected manually by reviewing electronic medical records and radiology information systems or calculated from the amount of contrast medial used for the CT scans. The application of the iterative reconstruction and its strength and use of the automatic kilovoltage control protocols for pediatric CT examinations were surveyed per institution.

\section{Data Processing and Data Retrieving}

CT scans were transferred to commercial dose management software (Radimetrics, Bayer Healthcare, Leverkusen, Germany) to retrieve the institution name, CT scanner model, scan region, age, sex, weight, peak kilovoltage $(k V p)$, effective diameter, and DRL quantities, including volume CT dose index (CTDI ${ }_{\text {vol }}$ ) and dose-length product (DLP). The effective dose was automatically calculated with Radimetrics software, with tissue weighting factors from ICRP publication 103 [12]. In our study, the reference phantom size of DRL quantities of abdominopelvic and chest CT examinations was $32 \mathrm{~cm}$; therefore, dose metrics based on the $16-\mathrm{cm}$ phantom size were converted to $32-\mathrm{cm}$ phantom-based values by multiplying with a factor of 0.5 . The measurement of the effective diameter, calculation of size-specific dose estimates (SSDE), and outlier detection are described in Supplementary Materials.

\section{Grouping of Patients}

First, we adopted weight bands from previously published 
guidelines for establishing DRLs for pediatric CT scans $[10,13]$. Weight bands were grouped as follows: $5 \mathrm{~kg},<5$ $\mathrm{kg} ; 15 \mathrm{~kg}, 5$ to $<15 \mathrm{~kg} ; 30 \mathrm{~kg}, 15$ to $<30 \mathrm{~kg}, 50 \mathrm{~kg}, 30$ to $<50 \mathrm{~kg}$; and $80 \mathrm{~kg}, 50$ to $<80 \mathrm{~kg}$. Second, data were grouped by using effective diameter as follows: $<13 \mathrm{~cm}$; $14-16 \mathrm{~cm} ; 17-20 \mathrm{~cm} ; 21-24 \mathrm{~cm}$; and $>24 \mathrm{~cm}$. Since previously published DRLs with grouping of data by the body size used the lateral dimensions, 15, 19, 24, and 29 $\mathrm{cm}$, the equivalent effective diameter was approximated from the American Association of Physicists in Medicine (AAPM) 204 report [14-17]. Third, age was used for grouping of dose data for comparison with other DRLs as follows: $0,<1$ month; 1 year, 1 month to $<1$ year; 5 years, 1 to $<5$ years; 10 years, 5 to $<10$ years, and 15 years, 10 to $<15$ years.

\section{Setting of DRL}

The median values of each DRL quantity for each group and hospital were calculated and defined as typical values [10]. Subsequently, the local DRL was set at the $75 \%$ value of the distribution of each hospital's typical values. This approach was recommended by both the ICRP and European guidelines $[10,13]$. Typical values were excluded if the number of examinations was fewer than five in each group for calculation of the DRL. Moreover, the DRL was set at the $75 \%$ value of the pooled distribution of entire institutions when the number of typical values in a group was less than five. $C_{T D I_{v o l}, S S D E}$, and DLP were used for DRL quantities. Distribution of the effective dose was modeled for pooled distribution after removing the upper and lower $5 \%$ values in each weight and age band.

Table 1. Patient Groups

\begin{tabular}{|c|c|c|c|c|c|c|c|c|c|c|}
\hline & \multicolumn{4}{|c|}{ Age } & \multicolumn{3}{|c|}{ Effective Diameter } & \multicolumn{3}{|c|}{ SSDE Conversion Factor } \\
\hline & Count & Median & Minimum & Maximum & Median & Minimum & Maximum & Median & Minimum & Maximum \\
\hline \multicolumn{11}{|c|}{ Abdomen } \\
\hline \multicolumn{11}{|c|}{ Weight, kg } \\
\hline 5 & 17 & 0.1 & 0 & 0.7 & 11 & 9 & 14 & 2.47 & 2.22 & 2.66 \\
\hline 15 & 259 & 2 & 0.2 & 7 & 15 & 12 & 18 & 2.14 & 1.91 & 2.38 \\
\hline 30 & 520 & 6.4 & 1.9 & 15.9 & 17 & 13 & 22 & 1.98 & 1.65 & 2.30 \\
\hline 50 & 496 & 11.1 & 3.9 & 16 & 21 & 12 & 28 & 1.71 & 1.32 & 2.38 \\
\hline 80 & 333 & 13.7 & 9 & 17 & 24 & 19 & 30 & 1.53 & 1.23 & 1.84 \\
\hline \multicolumn{11}{|l|}{ Chest } \\
\hline \multicolumn{11}{|c|}{ Weight, kg } \\
\hline 5 & 28 & 0.1 & 0 & 0.4 & 11 & 9 & 13 & 2.47 & 2.3 & 2.66 \\
\hline 15 & 245 & 1.7 & 0.1 & 8.3 & 14 & 11 & 17 & 2.22 & 1.98 & 2.47 \\
\hline 30 & 292 & 6 & 1.4 & 15.5 & 17 & 13 & 29 & 1.98 & 1.28 & 2.30 \\
\hline 50 & 221 & 11.3 & 5.8 & 15.8 & 22 & 14 & 26 & 1.65 & 1.43 & 2.22 \\
\hline 80 & 83 & 14 & 9.7 & 15.9 & 25 & 22 & 34 & 1.48 & 1.06 & 1.65 \\
\hline
\end{tabular}

SSDE = size-specific dose estimates
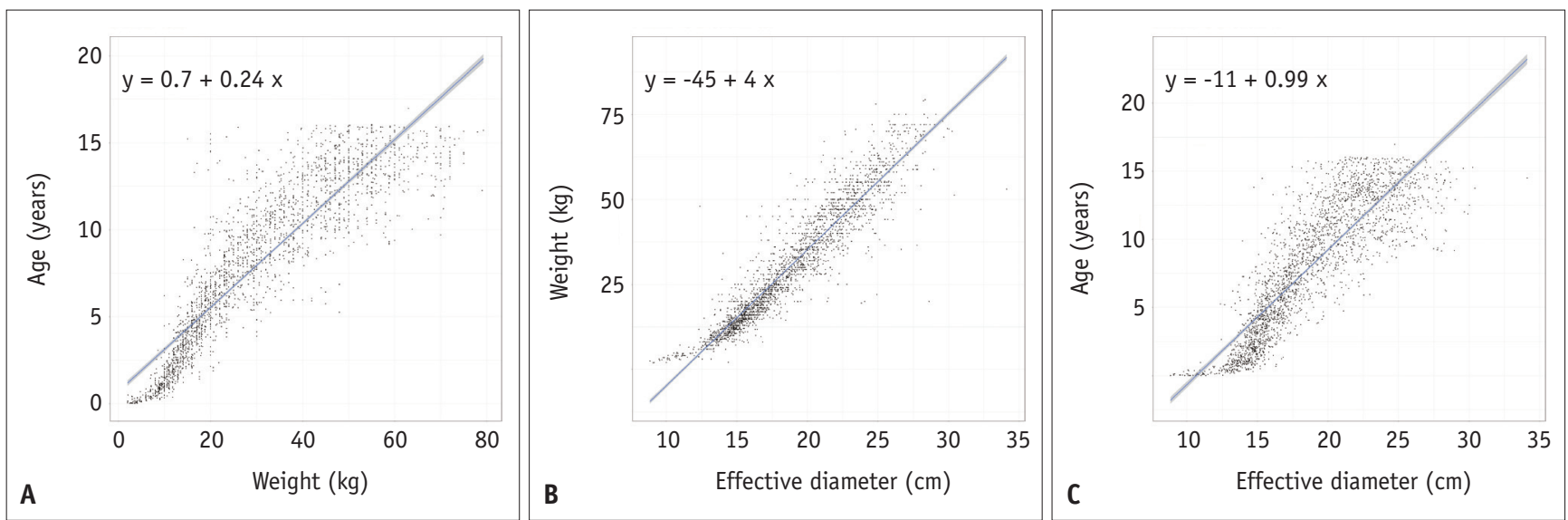

Fig. 1. Scatter plots of weight by age (A), effective diameter by weight (B), and effective diameter by age (C). 

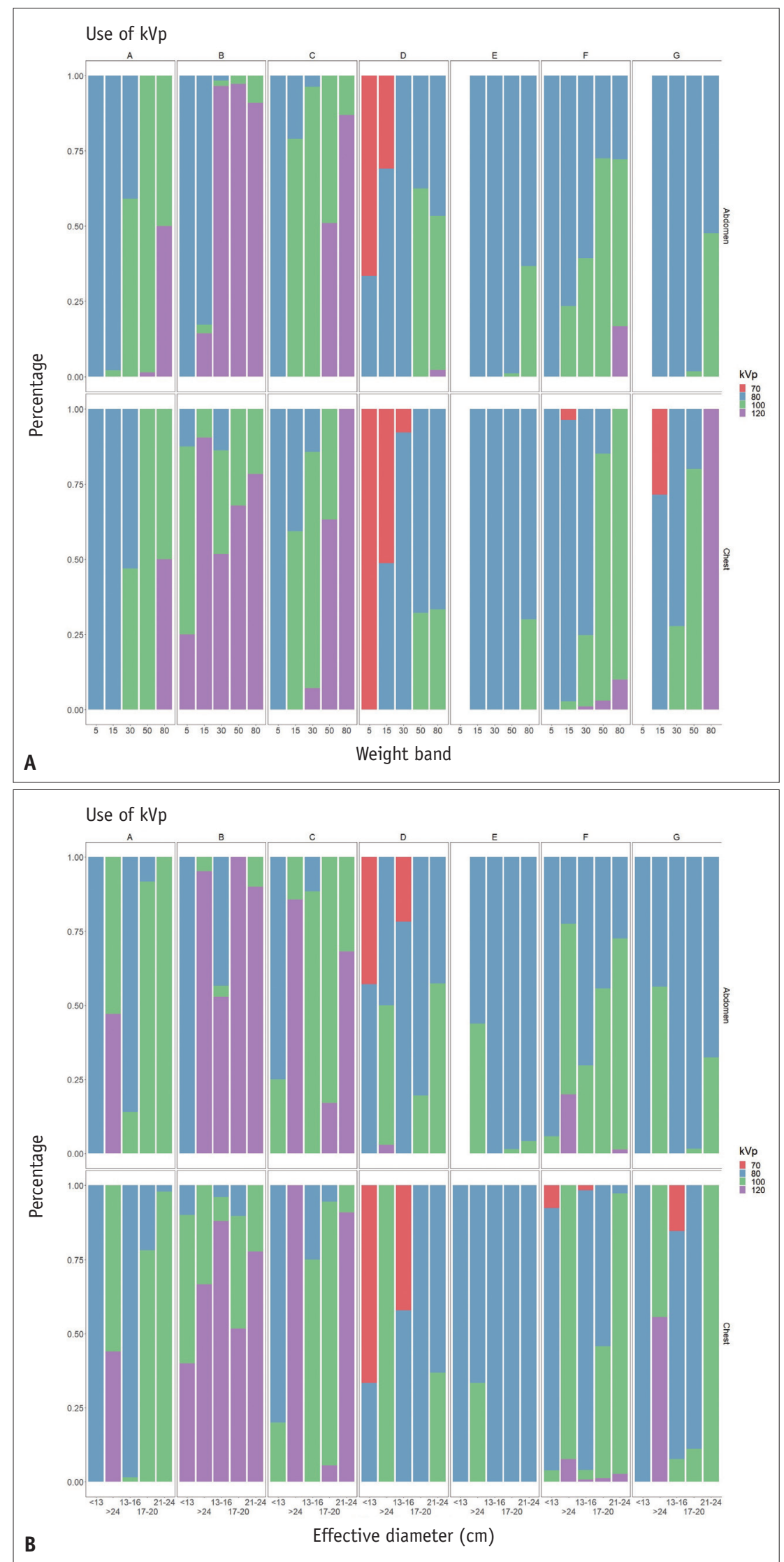

Fig. 2. Distributions of $k V p$ by the (A) weight band and (B) effective diameter. $k V p=$ peak kilovoltage 


\section{Statistical Analysis}

Descriptive statistics included the median, minimum, maximum, 25th percentile, and 75th percentile for each DRL quantity. These descriptive statistics were analyzed for each variable according to the institution, scan region, weight, effective diameter, and age. The linear-by-linear association test was performed to evaluate the relationship between the $k V p$ and the body weight and between the $k V p$ and the effective diameter. Statistical analyses were performed using MedCalc Statistical Software version 19.2.1 (MedCalc Software Ltd.) and IBM SPSS Statistics for Windows version 25.0 (IBM Corp.). A $p$ value $<0.05$ was considered statistically significant.

\section{RESULTS}

A total of 2909 CT examinations were collected retrospectively from nine university hospitals and uploaded to the automatic dose management system. Automatic analyses of the radiation dose failed in 64 CT examinations for the following reasons: $35 \mathrm{CT}$ examinations from five hospitals were not recognized by the automated dose management system, and 29 CT examinations from five hospitals had insufficient CT images. Finally, a total of 2845 CT examinations were valid for the dose analysis. Although the effective diameter was not automatically retrieved in 215 examinations from one hospital, it was measured manually.

Among the 2845 valid CT examinations, 226 from two institutions without information on the body weight, 35 with body weight $>80 \mathrm{~kg}$, and 24 dual-energy ones were excluded. Twelve CT examinations with an inappropriate age for the weight band and $54 \mathrm{CT}$ examinations with extreme DRL quantities were excluded during the outlier detection.

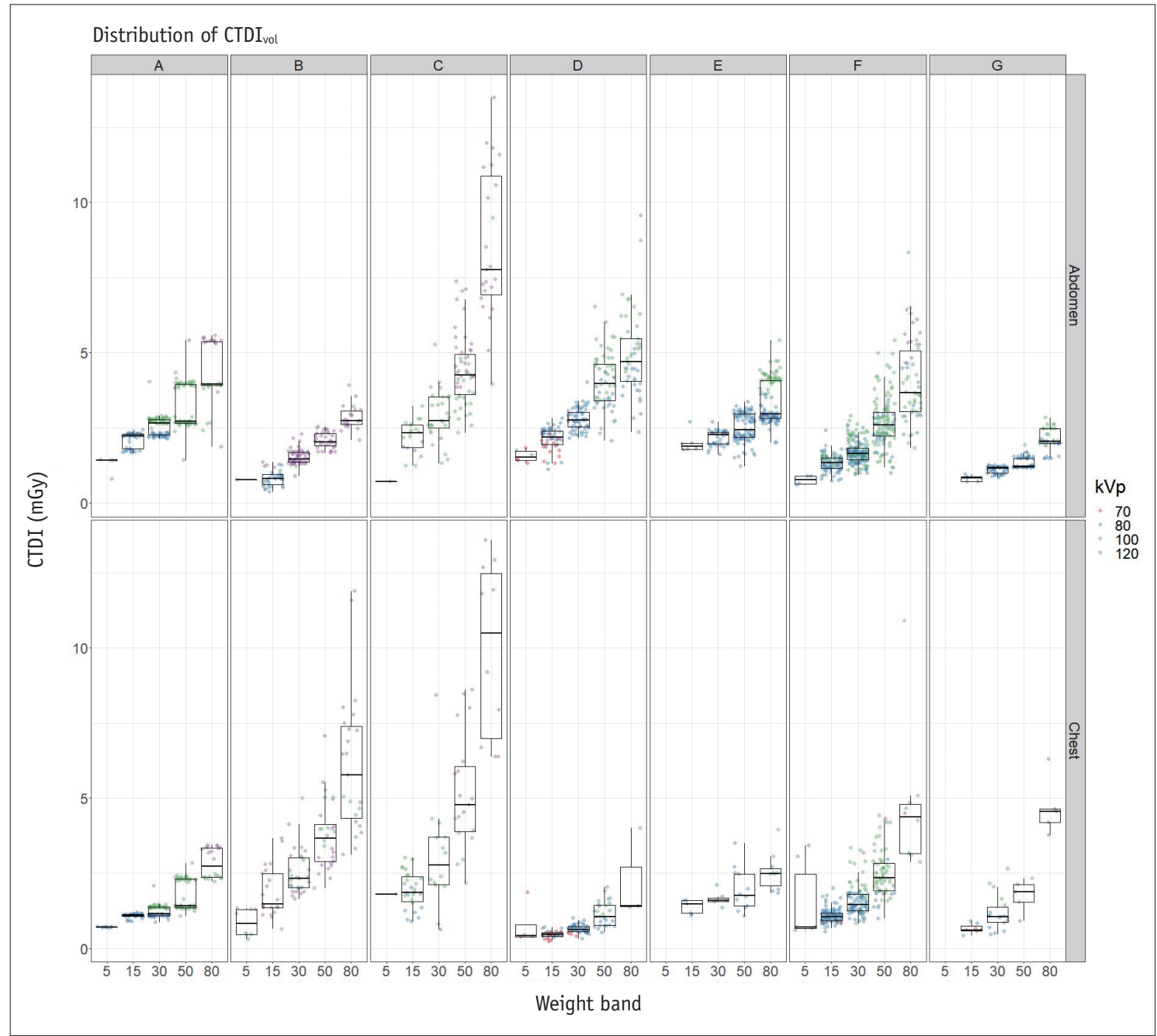

Fig. 3. Distribution of the CTDI $_{\text {vol }}$ by the weight band in each institution. The reference phantom size is $32 \mathrm{~cm}$. CTDI $I_{\text {vol }}=$ volume $C T$ dose index, $\mathrm{kVp}=$ peak kilovoltage 
Finally, 2494 CT examinations (1625 abdominopelvic and 869 chest (T examinations) from seven institutions were enrolled for the dose analysis.

Table 1 shows the patient age, effective diameter, and conversion factor for SSDE categorized with weight bands. Figure $1 \mathrm{~A}$ represents a scatter plot for the body weight by age. The body weight strongly correlated with age (Kendall's tau-b; 0.77, $p<0.01$ ). Figure $1 \mathrm{~B}$ and $\mathrm{C}$ show a scatter plot of the effective diameter for the body weight and age, respectively. The effective diameter strongly correlated with the body weight (Kendall's tau-b; 0.82, $p<$ 0.01 ) and moderately correlated with age (Kendall's tau-b; $0.7, p<0.01$ ).

All institutions used iterative reconstruction for both abdominopelvic and chest CT scans. The strengths of the iterative reconstruction were low, medium, and high at one, five, and one institution, respectively. Tube current modulation was applied for $88 \%$ of abdominopelvic CT scans and $93 \%$ of chest CT scans. All CT examinations were performed using multi-detector row CT scanners equipped with more than 64 channels.

Figure 2 shows the distribution of $k V p$ in each weight and effective diameter band. The range of $\mathrm{kVp}$ was from 70 to 120; institutions D, F, and G used $70 \mathrm{kVp}$ for small children. The kVp showed statistically significant linear-by-linear associations with the both weight and effective diameter in both abdominopelvic and chest CT examinations $(p<0.01)$. Institutions $\mathrm{D}, \mathrm{F}$, and $\mathrm{G}$ used the automatic $\mathrm{kV}$ control for pediatric body $\mathrm{CT}$ examinations.

Supplementary Tables 1 and 2 summarize the CTDI vol, DLP, $_{\text {, }}$ and SSDE of the abdominopelvic and chest CT examinations with weight bands. Figures 3, 4, and 5 represent the distributions of the CTDI $\mathrm{vol}_{\mathrm{v}}, \mathrm{SSDE}$, and DLP in each weight band.

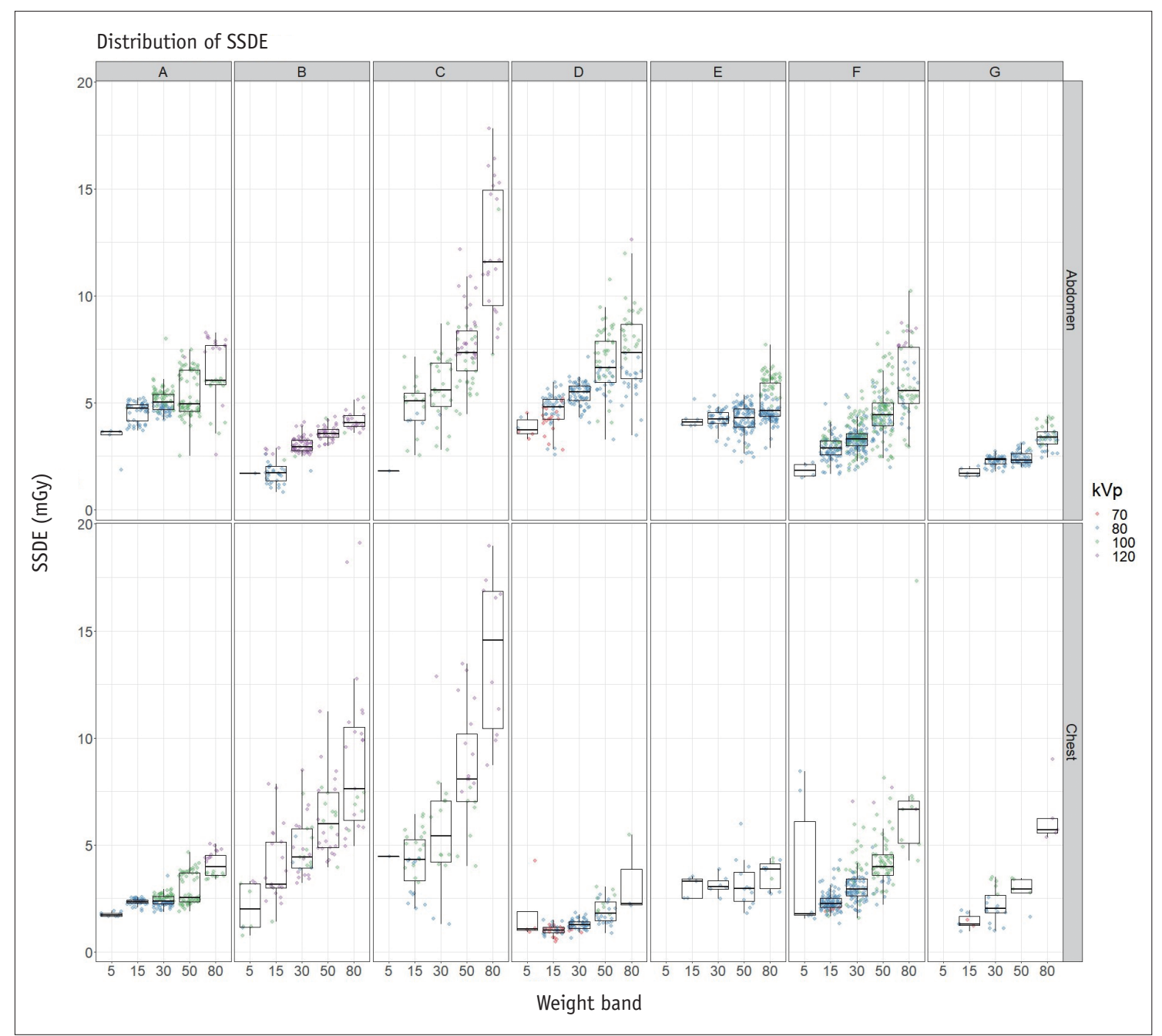

Fig. 4. Distribution of the SSDE by the weight band in each institution. $k V p=$ peak kilovoltage, SSDE = size-specific dose estimates 
Summaries of SSDE in effective diameters are shown in Supplementary Tables 3 and 4. Figure 6 represents a scatter plot of the CTDI vol by SSDE. The SSDE in all weight bands was greater than the CTDI ${ }_{\text {vol }}$ for both abdominopelvic and chest CT. Supplementary Tables 5 and 6 show the distribution of DRL quantities in age bands.

Tables 2 and 3 represent the local DRL for each weight band and effective diameter for both abdominopelvic and chest CT. The DRLs based on the age bands are shown in Supplementary Table 7. Since the sample sizes of the weight band in weight of $5 \mathrm{~kg}$, effective diameter $<13 \mathrm{~cm}$, and age band of 0 years were insufficient to set DRLs from the typical values, those DRLs were set from the pooled distribution of entire institutions.

Table 4 shows the distribution of effective doses. A comparison of DRLs obtained from this study with published DRLs is shown in Table 5 [9,14-16,18-21]. In our study, for the body weight of 5, 15, 30,50, and 80 $\mathrm{kg}$, CTDI $\mathrm{vol}_{\text {vol }}$ DRLs were 1.4, 2.2, 2.7, 4.0, and $4.7 \mathrm{mGy}$, respectively, for abdominopelvic $\mathrm{CT}$ and 1.2, 1.5, 2.3, 3.7, and $5.8 \mathrm{mGy}$, respectively, for chest CT. In European DRL, for the body weight of $5,15,30,50$, and $80 \mathrm{~kg}$, CTDI $_{\text {vol }}$ of DRLs were 3.5, 5.4, 7.3, and $18 \mathrm{mGy}$, respectively, for abdominopelvic CT and 1.4, 1.8, 2.7, 3.7, and 5.4, respectively, for chest CT [9]. DRLs were generally lower than those from other countries for abdominopelvic CT. However, DRLs of chest CT were slightly higher than those of the United States and Europe.

\section{DISCUSSION}

Our study was aimed at establishing local DRLs for pediatric body CT examinations from several university hospitals in South Korea. This is the first multicenter dose

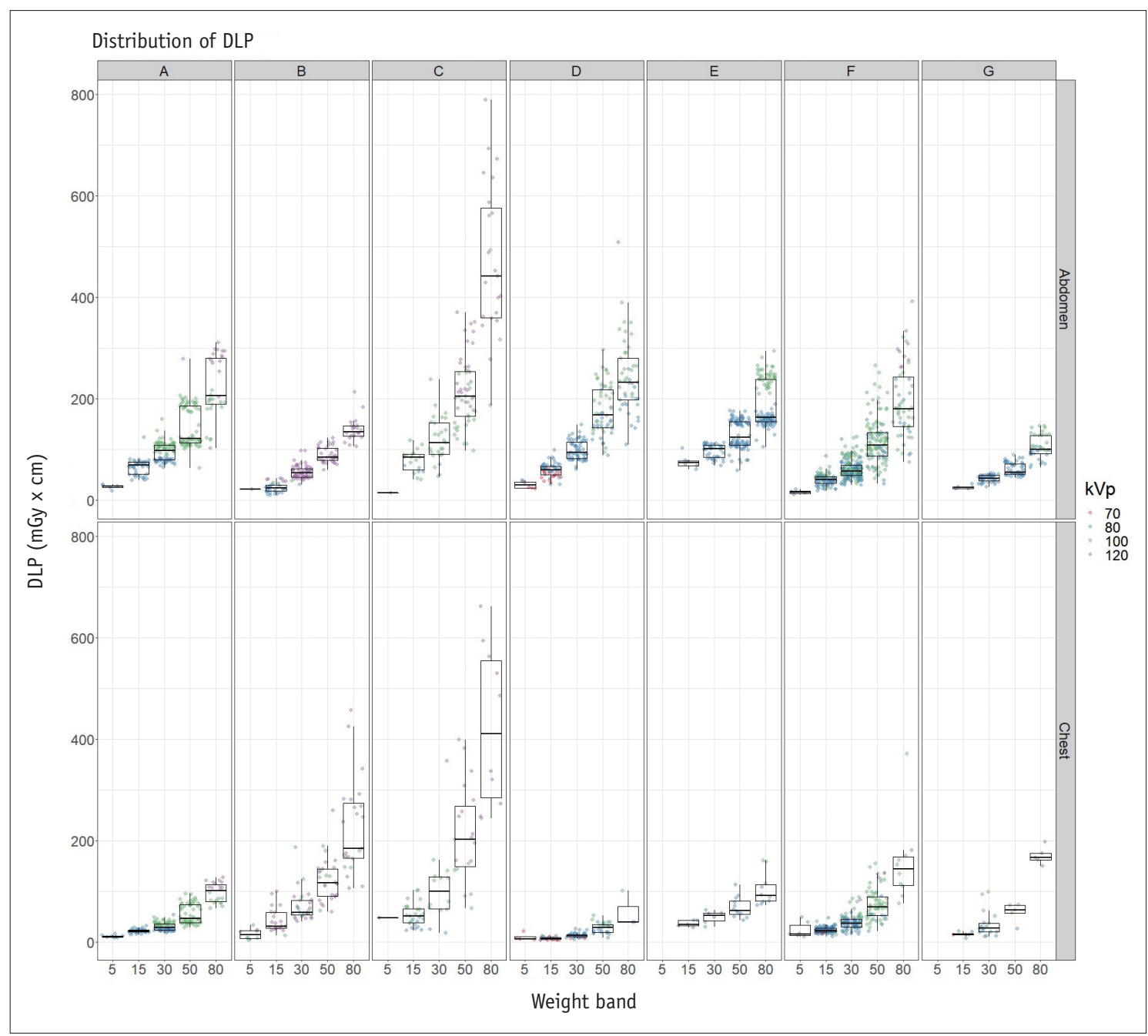

Fig. 5. Distribution of the DLP by the weight band in each institution. $D L P=$ dose-length product, $k V p=$ peak kilovoltage 


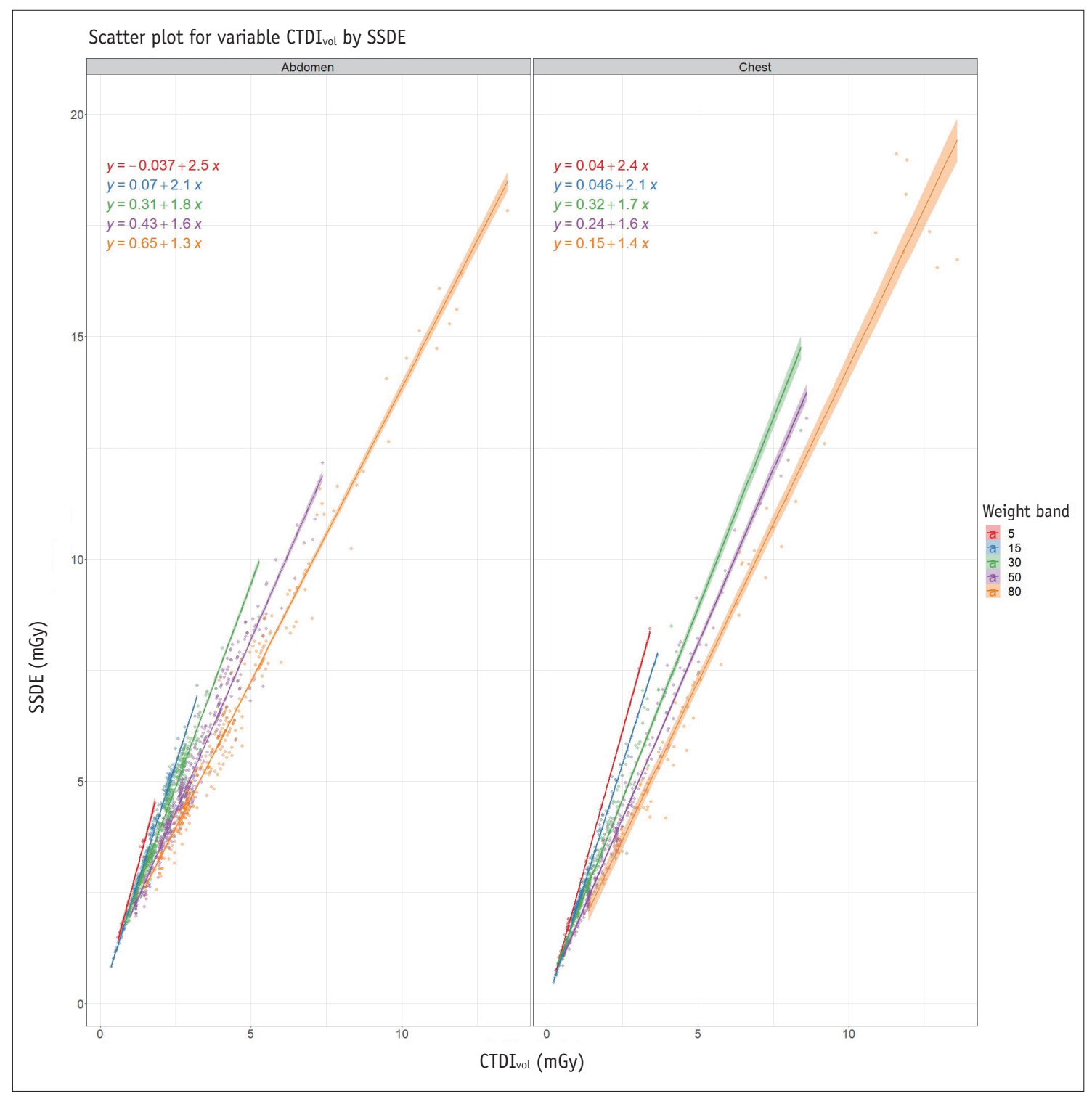

Fig. 6. Scatter plot of the CTDI $_{\text {vol }}$ by the SSDE. CTDI $_{\text {vol }}=$ volume CT dose index, SSDE = size-specific dose estimates

survey of pediatric CT examinations in South Korea, with grouping of patients by the body weight and size. There are various classes of DRL according to the target group of the dose survey, including typical, local, national, and regional DRLS [10]. Typical values represent the median value of the distribution in a healthcare facility or X-ray unit that is used to identify $X$-ray units requiring further optimization. The local DRL is typically set at the third quartile of the typical values of several healthcare facilities or X-ray units; thus, the DRL value in our study was a local DRL for a pediatric body CT examination. The national DRL should be based on most healthcare facilities across an entire country, and regional DRL is based on the DRL values of several countries within one geographic region or continent.
This study was an update of a previously published dose survey conducted in 2015 [21]. DRL values for both abdominopelvic and chest CT examinations were lower than the previous dose survey. Moreover, DRL values in this study were generally lower than those of other countries. Nevertheless, DRL values for chest CT examinations of larger patients were slightly higher than those in the United States and Europe, indicating the need for reviewing and optimizing CT protocols.

A different sampling of dose data may primarily affect differences in DRL values between the present and previous studies conducted in Korea. This study collected CT scans from several university hospitals where pediatric CT scans are performed frequently. It is more likely that designing 
and optimizing $\mathrm{CT}$ protocols are well managed by pediatric radiologists in these university hospitals. In our study, all institutions routinely applied iterative reconstruction and modern technologies, such as automatic tube current modulation. Moreover, several hospitals used the automatic kV control to optimize their CT protocols. However, the previous study sampled dose data from various hospitals that referred to a single tertiary hospital [21]. Thus, CT examinations from many hospitals where pediatric $\mathrm{X}$-ray procedures are performed infrequently or with suboptimal pediatric $\mathrm{CT}$ scan protocols might have been included.

Furthermore, $\mathrm{CT}$ examinations were performed with modern CT scanners equipped with more than 64 channels, while the previous study included examinations performed with

Table 2. Local Diagnostic Reference Levels of Abdominopelvic and Chest CT for Weight Bands

\begin{tabular}{|c|c|c|c|c|c|c|c|c|c|c|}
\hline & \multicolumn{4}{|c|}{$\mathrm{CTDI}_{\mathrm{vol}}$} & \multicolumn{3}{|c|}{ SSDE } & \multicolumn{3}{|c|}{ DLP } \\
\hline & Count & $\begin{array}{c}\text { Percentile } \\
75\end{array}$ & Median & $\begin{array}{c}\text { Percentile } \\
25\end{array}$ & $\begin{array}{c}\text { Percentile } \\
75\end{array}$ & Median & $\begin{array}{c}\text { Percentile } \\
25\end{array}$ & $\begin{array}{c}\text { Percentile } \\
75\end{array}$ & Median & $\begin{array}{c}\text { Percentile } \\
25\end{array}$ \\
\hline \multicolumn{11}{|l|}{ Scan } \\
\hline \multicolumn{11}{|c|}{ Abdominopelvic } \\
\hline \multicolumn{11}{|c|}{ Weight, kg } \\
\hline 5 & $17^{*}$ & 1.4 & 1.4 & 0.8 & 3.7 & 3.5 & 1.8 & 30.0 & 24.1 & 17.7 \\
\hline 15 & 7 & 2.2 & 1.9 & 0.8 & 4.8 & 4.1 & 1.7 & 73.8 & 59.9 & 25.6 \\
\hline 30 & 7 & 2.7 & 2.3 & 1.5 & 5.5 & 4.2 & 2.9 & 101.2 & 94.1 & 54.3 \\
\hline 50 & 7 & 4.0 & 2.6 & 2.0 & 6.6 & 4.4 & 3.6 & 168.8 & 121.5 & 84.5 \\
\hline 80 & 7 & 4.7 & 3.7 & 2.7 & 7.3 & 5.6 & 4.1 & 233.0 & 179.8 & 135.5 \\
\hline \multicolumn{11}{|l|}{ Chest } \\
\hline \multicolumn{11}{|c|}{ Weight, kg } \\
\hline 5 & $28^{*}$ & 1.2 & 0.7 & 0.6 & 3.0 & 1.7 & 1.4 & 21.5 & 12.1 & 8.9 \\
\hline 15 & 7 & 1.5 & 1.1 & 0.6 & 3.3 & 2.3 & 1.3 & 34.6 & 23.4 & 14.0 \\
\hline 30 & 7 & 2.3 & 1.5 & 1.1 & 4.4 & 2.9 & 2.0 & 59.0 & 37.5 & 28.0 \\
\hline 50 & 7 & 3.7 & 1.9 & 1.4 & 6.0 & 2.9 & 2.5 & 117.4 & 63.0 & 46.5 \\
\hline 80 & 6 & 5.8 & 4.5 & 2.7 & 7.6 & 6.2 & 4.0 & 184.4 & 155.6 & 101.1 \\
\hline
\end{tabular}

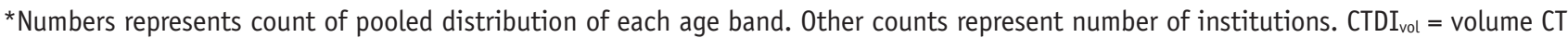
dose index, DLP = dose-length product, $\mathrm{SSDE}=$ size-specific dose estimates

Table 3. Local Diagnostic Reference Levels of Abdominopelvic and Chest CT for Effective Diameter Bands

\begin{tabular}{|c|c|c|c|c|c|c|c|c|c|c|}
\hline & \multicolumn{4}{|c|}{ SSDE } & \multicolumn{3}{|c|}{$\mathrm{CTDI}_{\mathrm{vol}}$} & \multicolumn{3}{|c|}{ DLP } \\
\hline & Count & $\begin{array}{c}\text { Percentile } \\
75\end{array}$ & Median & $\begin{array}{c}\text { Percentile } \\
25\end{array}$ & $\begin{array}{c}\text { Percentile } \\
75\end{array}$ & Median & $\begin{array}{c}\text { Percentile } \\
25 \\
\end{array}$ & $\begin{array}{c}\text { Percentile } \\
75 \\
\end{array}$ & Median & $\begin{array}{c}\text { Percentile } \\
25\end{array}$ \\
\hline \multicolumn{11}{|l|}{ Scan } \\
\hline \multicolumn{11}{|l|}{ Abdomen } \\
\hline \multicolumn{11}{|c|}{ Effective diameter, cm } \\
\hline$<13$ & $61^{*}$ & 4.1 & 3.5 & 2.1 & 1.8 & 1.4 & 0.9 & 46.2 & 32.0 & 21.5 \\
\hline $14-16$ & 7 & 5.0 & 4.0 & 2.6 & 2.3 & 1.9 & 1.3 & 79.0 & 66.5 & 41.9 \\
\hline $17-20$ & 7 & 5.7 & 4.1 & 3.2 & 3.0 & 2.2 & 1.7 & 112.6 & 107.6 & 66.2 \\
\hline $21-24$ & 7 & 7.1 & 4.8 & 3.8 & 4.3 & 2.9 & 2.3 & 190.5 & 153.9 & 104.2 \\
\hline$>24$ & 7 & 7.2 & 6.0 & 4.1 & 5.0 & 3.9 & 2.7 & 238.2 & 201.4 & 132.4 \\
\hline \multicolumn{11}{|l|}{ Chest } \\
\hline \multicolumn{11}{|c|}{ Effective diameter, cm } \\
\hline$<13$ & $73^{*}$ & 2.8 & 2.0 & 1.6 & 1.2 & 0.9 & 0.6 & 23.6 & 17.9 & 9.3 \\
\hline $14-16$ & 7 & 4.6 & 2.4 & 1.8 & 2.2 & 1.1 & 0.8 & 50.0 & 25.9 & 20.0 \\
\hline $17-20$ & 7 & 4.3 & 3.0 & 1.9 & 2.3 & 1.6 & 1.0 & 62.0 & 45.2 & 27.0 \\
\hline $21-24$ & 7 & 5.3 & 3.5 & 3.0 & 3.3 & 2.1 & 1.8 & 111.4 & 71.3 & 55.9 \\
\hline$>24$ & 7 & 7.5 & 5.4 & 3.4 & 5.0 & 3.8 & 2.4 & 180.0 & 119.7 & 85.4 \\
\hline
\end{tabular}

*Numbers represents count of pooled distribution of each age band. Other counts represent number of institutions. CTDI $_{\text {vol }}=$ volume $\mathrm{CT}$ dose index, DLP = dose-length product, $\mathrm{SSDE}=$ size-specific dose estimates 
Table 4. Distribution of the Effective Dose for Each Weight and Age Band

\begin{tabular}{|c|c|c|c|c|c|c|}
\hline & \multicolumn{6}{|c|}{ Effective Dose } \\
\hline & Count & Mean & Standard Deviation & Median & Percentile 25 & Percentile 75 \\
\hline \multicolumn{7}{|l|}{ Scan } \\
\hline \multicolumn{7}{|c|}{ Abdominopelvic } \\
\hline \multicolumn{7}{|c|}{ Weight, kg } \\
\hline 5 & 17 & 2.4 & 0.9 & 2.6 & 1.4 & 3.0 \\
\hline 15 & 259 & 2.7 & 1.1 & 2.6 & 1.8 & 3.7 \\
\hline 30 & 520 & 2.7 & 1.0 & 2.5 & 2.0 & 3.4 \\
\hline 50 & 496 & 3.2 & 1.3 & 3.0 & 2.4 & 3.9 \\
\hline 80 & 333 & 4.1 & 2.0 & 3.4 & 2.9 & 4.8 \\
\hline \multicolumn{7}{|l|}{ Chest } \\
\hline \multicolumn{7}{|c|}{ Weight, kg } \\
\hline 5 & 28 & 1.7 & 1.3 & 1.2 & 1.0 & 2.3 \\
\hline 15 & 245 & 1.6 & 1.0 & 1.4 & 1.1 & 1.8 \\
\hline 30 & 292 & 1.6 & 1.1 & 1.4 & 1.0 & 1.9 \\
\hline 50 & 221 & 2.3 & 1.7 & 1.9 & 1.2 & 2.7 \\
\hline 80 & 83 & 4.2 & 2.8 & 3.1 & 2.3 & 5.4 \\
\hline \multicolumn{7}{|l|}{ Scan } \\
\hline \multicolumn{7}{|c|}{ Abdominopelvic } \\
\hline \multicolumn{7}{|c|}{ Age, years } \\
\hline 0 & 9 & 2.4 & 0.9 & 2.6 & 1.4 & 3.0 \\
\hline 1 & 75 & 2.8 & 1.2 & 2.9 & 1.6 & 3.8 \\
\hline 5 & 348 & 2.7 & 1.0 & 2.4 & 1.9 & 3.4 \\
\hline 10 & 484 & 2.9 & 1.2 & 2.8 & 2.0 & 3.5 \\
\hline 15 & 709 & 3.6 & 1.7 & 3.1 & 2.7 & 4.2 \\
\hline \multicolumn{7}{|l|}{ Chest } \\
\hline \multicolumn{7}{|c|}{ Age, years } \\
\hline 0 & 13 & 1.4 & 0.8 & 1.2 & 0.8 & 1.3 \\
\hline 1 & 64 & 1.7 & 1.2 & 1.4 & 0.7 & 2.2 \\
\hline 5 & 288 & 1.5 & 0.9 & 1.4 & 1.1 & 1.7 \\
\hline 10 & 238 & 1.8 & 1.2 & 1.5 & 1.1 & 2.1 \\
\hline 15 & 266 & 2.9 & 2.3 & 2.2 & 1.5 & 3.4 \\
\hline
\end{tabular}

older CT scanners with less than 16 channels. Improvement in hardware with better dose efficiency, dedicated automatic exposure control, and development of iterative reconstruction may substantially contribute to radiation dose reduction [22]. Therefore, care should be taken when comparing the results of this study with that of the previous dose survey.

Estimating and managing the dose from $\mathrm{X}$-ray procedures in pediatric patients are challenging. There is a wide variation because of the different body habitus with growth; thus, standardization of the patient in a single reference band is not possible, particularly for conventionally used age bands $[15,16,23]$. The patient's weight, cross-sectional area, or body dimension may be used as an alternative to age in optimizing CT protocols. In previous studies, the patient's individual size did not correlate with age; therefore, the authors suggested that patient size is preferable to age bands when designing protocols for imaging procedures and managing radiation doses $[10,24]$. Furthermore, as modern radiography, fluoroscopy, and CT scanners have equipped automatic exposure control systems or tube current modulation based on the attenuation of the regions of interest $[10,25,26]$, the grouping of the dose survey into the patient size or waterequivalent diameter should be considered for establishing DRLs in pediatric CT examinations.

However, the application of the body size and X-ray attenuation characteristics require additional workload and dedicated software, hindering their use in routine practice and dose surveys. In our study, all hospitals used CT protocols designed according to the patient's body weight, and none of the hospitals used the body size or X-ray attenuation characteristics. Few studies conducted dose 


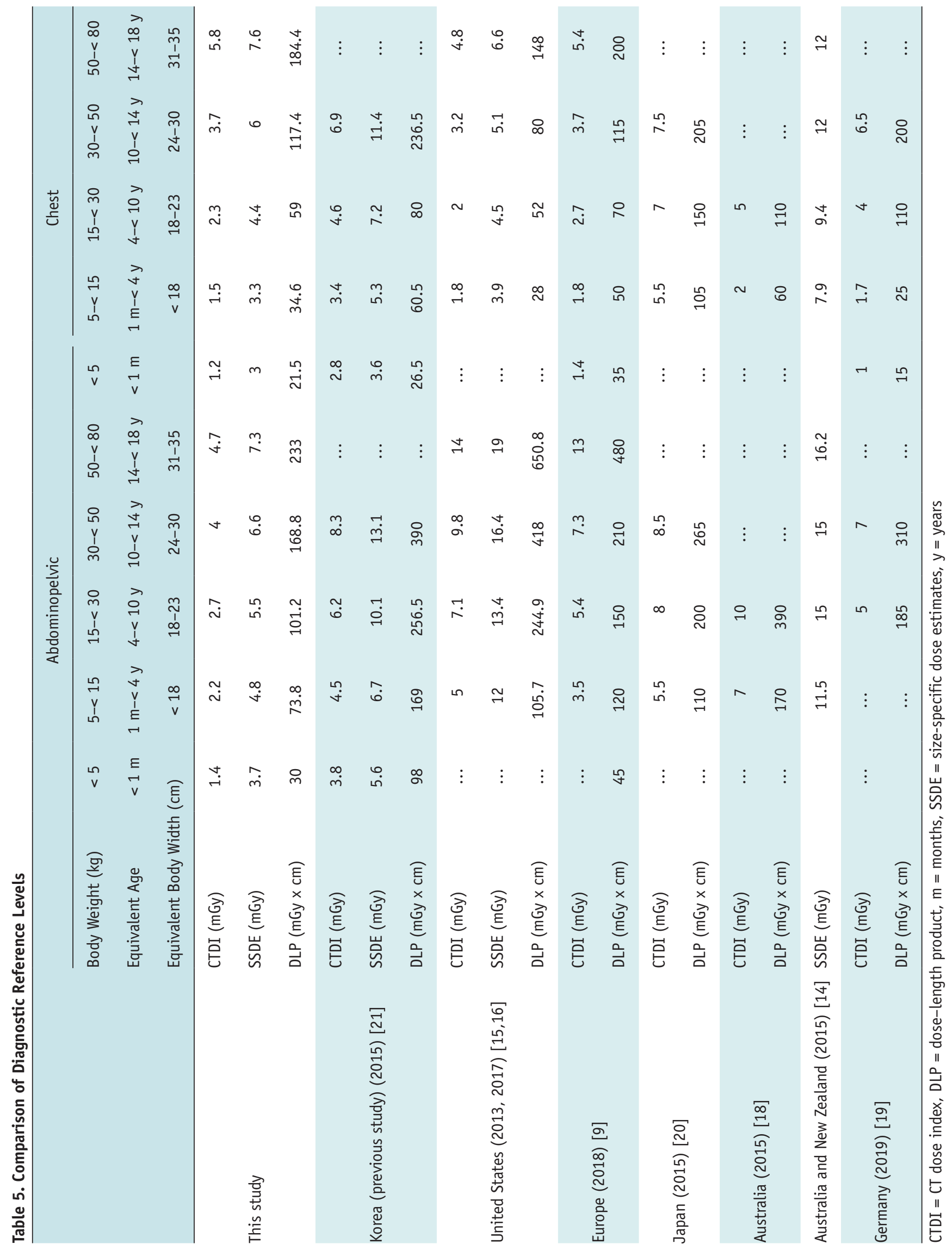


surveys for pediatric $\mathrm{CT}$ examinations by grouping patients according to the body size [14-16].

Therefore, current guidelines on establishing DRLs in pediatric CT scans recommend grouping of dose surveys into body weight groups instead of the classically used age bands $[10,13]$. In a previous study, the authors suggested grouping the survey data based on patient weight when a smaller sample is used and using age groups when the patient's weight is not known or with large sample sizes, particularly in less-developed countries [11]. According to previous studies, which achieved similar results as our study, the weight well-correlated with the body size $[20,27,28]$.

Our study had several limitations. First, data of neonates and infants are not sufficient to obtain typical values in some institutions, and therefore, DRLs were obtained from the pooled data. Although we collected data from one or two CT scanners that performed most pediatric CT examinations in a given period, some examinations in this age group were still challenging.

Second, patient weight was calculated from the amount of contrast agents used during the CT examination in six of seven hospitals because information on the body weight was not easily accessible in many hospitals. Although modern CT scanners can embed the body weight into the DICOM header, its current use seems limited in most institutions. Embedding of the variables that can be used for the analysis of the radiation dose, such as the weight, height, and X-ray attenuation characters into the DICOM header will facilitate automated, time-saving, and precise dose surveys in the future.

Third, care should be taken in interpreting the data of this study because this study was conducted based on university hospitals, which are likely to have well-optimized pediatric CT protocols. It is important to recognize that the DRL values of our study were local DRLs of several university hospitals and not the national DRLs.

Finally, we did not conduct structured image quality assessments for collected CT scans because of the large sample size. Pediatric radiologists confirmed that the CT scans we collected had clinically acceptable quality. Various factors, such as the age, clinical status, clinical indication, and the radiologist's personal preference, may affect determining acceptable image quality and optimizing CT protocols.

In conclusion, our study set local DRLs in pediatric abdominopelvic and chest CT examinations based on the weight and body size by using an automated dose collection system. The DRL values were decreased compared to the previous dose survey and values from other countries. Further studies with more facilities should be performed for the development of national DRLs and regular update of the current DRLs.

\section{Supplement}

The Supplement is available with this article at https://doi.org/10.3348/kjr.2020.0890.

\section{Conflicts of Interest}

The authors have no potential conflicts of interest to disclose.

\section{Acknowledgments}

We thanks Mr. JongWon Lee from Bayer Korea Ltd. for supporting set up an automated dose management software.

\section{Author Contributions}

Conceptualization: Jae-Yeon Hwang, Young Hun Choi. Data curation: Jae-Yeon Hwang, Young Hun Choi. Formal analysis: Jae-Yeon Hwang. Funding acquisition: Jae-Yeon Hwang. Investigation: Young Hun Choi, Hee Mang Yoon, Young Jin Ryu, Hyun Joo Shin, Hyun Gi Kim, So Mi Lee, Sun Kyung You, Ji Eun Park. Methodology: Young Hun Choi, Hee Mang Yoon. Project administration: Jae-Yeon Hwang. Visualization: Jae-Yeon Hwang. Writing - original draft: JaeYeon Hwang, Young Hun Choi. Writing-review \& editing: Hee Mang Yoon, Young Jin Ryu, Hyun Joo Shin, Hyun Gi Kim, So Mi Lee, Sun Kyung You, Ji Eun Park.

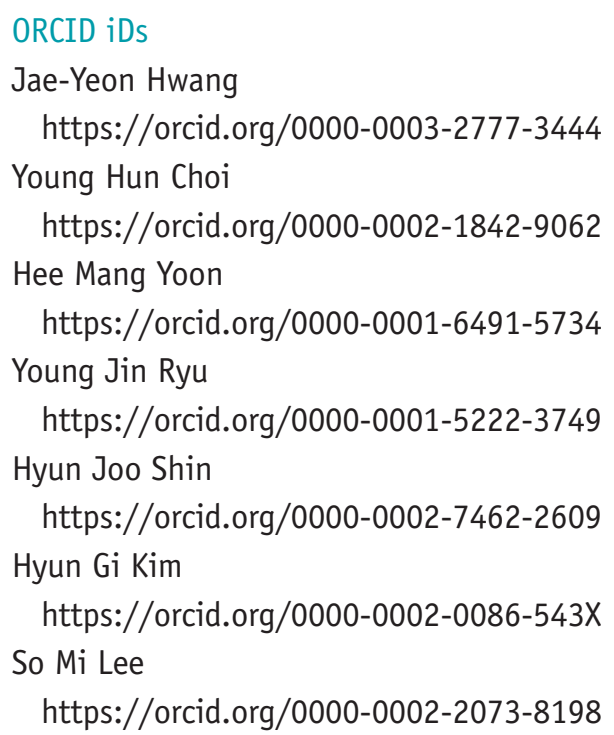


Sun Kyung You

https://orcid.org/0000-0002-1026-5809

Ji Eun Park

$$
\text { https://orcid.org/0000-0003-1305-0931 }
$$

\section{REFERENCES}

1. OECD. Health at a glance. Oecd-ilibrary.org Web site. https:// www.oecd-ilibrary.org/social-issues-migration-health/healthat-a-glance-2019_4dd50c09-en. Published 2019. Accessed February 23, 2020

2. Broder J, Warshauer DM. Increasing utilization of computed tomography in the adult emergency department, 2000-2005. Emerg Radiol 2006;13:25-30

3. Brody AS, Frush DP, Huda W, Brent RL; American Academy of Pediatrics Section on Radiology. Radiation risk to children from computed tomography. Pediatrics 2007;120:677-682

4. Bosch de Basea M, Salotti JA, Pearce MS, Muchart J, Riera L, Barber I, et al. Trends and patterns in the use of computed tomography in children and young adults in Catalonia results from the EPI-CT study. Pediatr Radiol 2016;46:119-129

5. Wildman-Tobriner B, Strauss KJ, Bhargavan-Chatfield M, Kadom N, Vock P, Applegate KE, et al. Using the American College of Radiology Dose Index Registry to evaluate practice patterns and radiation dose estimates of pediatric body CT. AJR Am J Roentgenol 2018;210:641-647

6. Chodick G, Ronckers C, Ron E, Shalev V. The utilization of pediatric computed tomography in a large Israeli Health Maintenance Organization. Pediatr Radiol 2006;36:485-490

7. Linet MS, Kim KP, Rajaraman P. Children's exposure to diagnostic medical radiation and cancer risk: epidemiologic and dosimetric considerations. Pediatr Radiol 2009;39 Suppl 1:S4-26

8. Anonymous. Radiological protection and safety in medicine. Annals of the ICRP 1996;26:1-31

9. European Comission. European guidelines on diagnostic reference levels for paediatric imaging. Luxembourg: EU Publications, 2018

10. Vañó E, Miller DL, Martin CJ, Rehani MM, Kang K, Rosenstein $M$, et al. ICRP publication 135: diagnostic reference levels in medical imaging. Ann ICRP 2017;46:1-144

11. Vassileva J, Rehani M. Patient grouping for dose surveys and establishment of diagnostic reference levels in paediatric computed tomography. Radiat Prot Dosimetry 2015;165:81-85

12. The 2007 recommendations of the international commission on radiological protection. Icrp.org Web site. https://www. icrp.org/publication.asp?id=ICRP\%20Publication\%20103. Published 2007. Accessed February 23, 2020

13. Granata C, Sorantin E, Seuri R, Owens CM. European Society of Paediatric Radiology Computed Tomography and Dose Task Force: European guidelines on diagnostic reference levels for paediatric imaging. Pediatr Radiol 2019;49:702-705
14. Jackson D, Atkin K, Bettenay F, Clark J, Ditchfield MR, Grimm JE, et al. Paediatric CT dose: a multicentre audit of subspecialty practice in Australia and New Zealand. Eur Radiol 2015;25:3109-3122

15. Goske MJ, Strauss KJ, Coombs LP, Mandel KE, Towbin AJ, Larson DB, et al. Diagnostic reference ranges for pediatric abdominal CT. Radiology 2013;268:208-218

16. Strauss KJ, Goske MJ, Towbin AJ, Sengupta D, Callahan MJ, Darge $\mathrm{K}$, et al. Pediatric chest CT diagnostic reference ranges: development and application. Radiology 2017;284:219-227

17. Boone J, Strauss K, Cody D, McCollough C, McNitt-Gray M, Toth T. The Report of AAPM Task Group 204: size-specific dose estimates (SSDE) in pediatric and adult body CT examinations. Alexandria: American Association of Physicists in Medicine, 2011

18. Wallace A, Hayton A, Thomas P, Beveridge T. The 2011-2013 national diagnostic reference level service report. ARPANSA Technical Report No. 171. Victoria: Australian Radiation Protection and Nuclear Safety Agency, 2015

19. Schegerer A, Loose R, Heuser LJ, Brix G. Diagnostic reference levels for diagnostic and interventional $\mathrm{X}$-ray procedures in Germany: update and handling. Rofo 2019;191:739-751

20. Imai R, Miyazaki 0, Horiuchi T, Kurosawa H, Nosaka S. Local diagnostic reference level based on size-specific dose estimates: assessment of pediatric abdominal/pelvic computed tomography at a Japanese national children's hospital. Pediatr Radiol 2015;45:345-353

21. Hwang JY, Do KH, Yang DH, Cho YA, Yoon HK, Lee JS, et al. A survey of pediatric CT protocols and radiation doses in South Korean hospitals to optimize the radiation dose for pediatric CT scanning. Medicine (Baltimore) 2015;94:e2146

22. Kubo T. Vendor free basics of radiation dose reduction techniques for CT. Eur J Radiol 2019;110:14-21

23. WHO Multicentre Growth Reference Study Group. WHO Child Growth Standards based on length/height, weight and age. Acta Paediatr Suppl 2006;450:76-85

24. Kleinman PL, Strauss KJ, Zurakowski D, Buckley KS, Taylor GA. Patient size measured on CT images as a function of age at a tertiary care children's hospital. AJR Am J Roentgenol 2010;194:1611-1619

25. McCollough CH, Bruesewitz MR, Kofler JM Jr. CT dose reduction and dose management tools: overview of available options. Radiographics 2006;26:503-512

26. Kalra MK, Maher MM, Toth TL, Schmidt B, Westerman BL, Morgan HT, et al. Techniques and applications of automatic tube current modulation for CT. Radiology 2004;233:649-657

27. Khawaja RD, Singh S, Vettiyil B, Lim R, Gee M, Westra S, et al. Simplifying size-specific radiation dose estimates in pediatric CT. AJR Am J Roentgenol 2015;204:167-176

28. Iriuchijima A, Fukushima Y, Nakajima T, Tsushima Y, Ogura A. Simple method of size-specific dose estimates calculation from patient weight on computed tomography. Radiat Prot Dosimetry 2018;178:208-212 